\title{
Neonatal Hypotension, AE
}

National Cancer Institute

\section{Source}

National Cancer Institute. Neonatal Hypotension, AE. NCI Thesaurus. Code C154930.

An adverse event in a newborn characterized by abnormally low blood pressure, which is usually symptomatic. 